\title{
Web-based Conceptual Design Support System
}

\author{
Dereje Engida Woldemichael ${ }^{1, ~ a ~}{ }^{*}$ and Fakhruldin Mohd Hashim ${ }^{1, b}$ \\ ${ }^{1}$ Universiti Teknologi PETRONAS, Mechanical Engineering Department, Bandar Seri Iskandar, \\ 31750 Tronoh, Perak, Malaysia

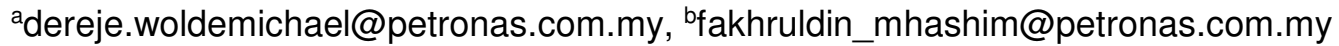

Keywords: Conceptual design, design support system, collaborative design, web-based system.

\begin{abstract}
Conceptual design is one of the most critical and important phase of design with least computer support. This paper presents an extension of a standalone conceptual design support tool developed previously by the authors on a web-based environment. The proposed framework is suitable for collaborative design which is performed by multiple participants, each potentially capable of proposing values for design issues and/or evaluating these choices. The framework uses design reuse philosophy which plays a major role in product development especially during the early concept generation phase supplementing the designer's knowledge by providing stored knowledge outside the designer's area of expertise. The reused design knowledge from existing product has an advantage of being tested for reliability in previous design and may inspire the designer to generate new concepts. Based on this philosophy, a web-based conceptual design support system (CDSS) has been developed and deployed on a local server which can be accessed on intranet network. The CDSS can be used either to generate new concepts from the design repository (database) or from the designer which can be added to the repository through knowledge acquisition module for future design.
\end{abstract}

\section{Introduction}

Conceptual design is one of the most critical and important phase of design with least computer support compared to the later phases of design. In an attempt to address this lack of computer aided conceptual design support tools, the authors had developed a standalone software known as CDST (conceptual design support tool) which can be installed on personal computers [1-3]. CDST is a conceptual design support system developed to assist designers in performing conceptual design process of mechanical products such as functional modelling (using standard vocabularies of functions), generating concepts (from database) and displaying on morphology chart, concept combination, and concept evaluation. Although the database is designed to expand with time, it is limited to single computer on which it is installed and difficult to share among designers in the same group.

Product design knowledge can be obtained from experienced designers, through proper knowledge acquisition system and from existing products using design reuse philosophy. This design knowledge can be made available through a collaborative product design information management system. In addition to obtaining the product data and knowledge from networked product design information management system, designers can also contribute new ideas to the system, which will help to build the design repository (database) for future use and train novice designers. As design is mostly a knowledge generation process, the intensive use of design knowledge and the capability to manage these knowledge is the key requirement for future intelligent CAD systems [4]. As pointed out by Goel et al. [5], the next generation of CAD will be defined by its cognitive account of design, support for collaborative design, conceptual design and creative design. The proposed web-based conceptual design support system is in line with this goal.

Conceptual design process is knowledge intensive, which requires collaboration of expertise from different disciplines. Design knowledge resides in the minds of experienced designers. This expertise may geographically be located at different parts of the world, but can contribute to the design if there is a suitable platform to do so. Furthermore, using design reuse philosophy, design knowledge from 
existing products can be used in future conceptual design process which makes the design process faster and cheaper. Design reuse plays a major role in product development especially during the early concept generation phase supplementing the designer's knowledge by providing stored knowledge outside the designer's area of expertise. The reused design knowledge from existing product has an advantage of being tested for reliability in previous design. The design knowledge can be used for similar application as in the original design or for completely different application. To get competative and inovative product, possible design options should be explored exhaustively and carefully to make necessary changes early in the design process. Based on this design philosophy, a web-based concpetual design support system is developed.

Although humans are creative in nature, they have limitation in processing large data. Because of this, it is difficult to explore all the design space within a given time and make sound judgment. On the other hand, computers are capable of handling and processing large data. This paper presents an extension of CDST to address this limitation and make it suitable for collaborative design environment through shared database. Accordingly, the proposed web-based conceptual design support system (CDSS) can be used to integrate human creativity with data handling capability of machines.

\section{Basics of Conceptual Design Process model}

Conceptual design process can be considered as the transformation of design specification, which is given as requirement list into one or more concepts that can satisfy these requirements for further development. A conceptual design process model that integrates a systematic design approach with a knowledge-based system is used to develop the CDSS [3]. In this model design problems can be described in terms of the overall function which is described as action verb and noun. The overall function is decomposed in to less complex and easily manageable elemental mechanical subfunctions which are also described in verb + noun format. The description and decomposition of the functions is entirely done by the designer assisted by function library which consist of vocabulary of mechanical functions derived from reconciled functional basis with function classification and flow classification [6]. In reconciled functional basis, functions are classified into eight primary classes: branch, channel, connect, control magnitude, convert, provision, signal and support. These primary classes are further classified into forty-five secondary and tertiary classes of action verbs. There are three basic (primary) classes of flows: material, energy and signal, which also have forty-two secondary and tertiary flows. Function can be represented by combining the primary, secondary or tertiary classes of functions and flows in functional basis.

Functional structure of a given design problem can be constructed from the functions in the elemental mechanical functions. For each subfunction, alternative concepts will be generated manually by the designer or by searching alternative concept database. The generated concepts can be evaluated at function level before design synthesis through concept combination process. Finally, the concept variants are evaluated using progressive concept selection process from which one or two concept variants are considered for testing and embodiment design.

\section{Framework of Web-based CDSS}

The framework of the proposed web-based CDSS is shown in Fig. 1. It starts with a hypothetical designer who has either design problem to be solved or new concept/idea in mind that can be used to address certain functions. The representation of design knowledge follows standard mechanical function representation adopted from reconciled functional basis [6]. The framework is suitable for collaborative design which is performed by multiple participants, each potentially capable of proposing values for design issues and/or evaluating these choices from their own particular perspective. The designers who might be located in different parts of the world can contribute in updating the database. 


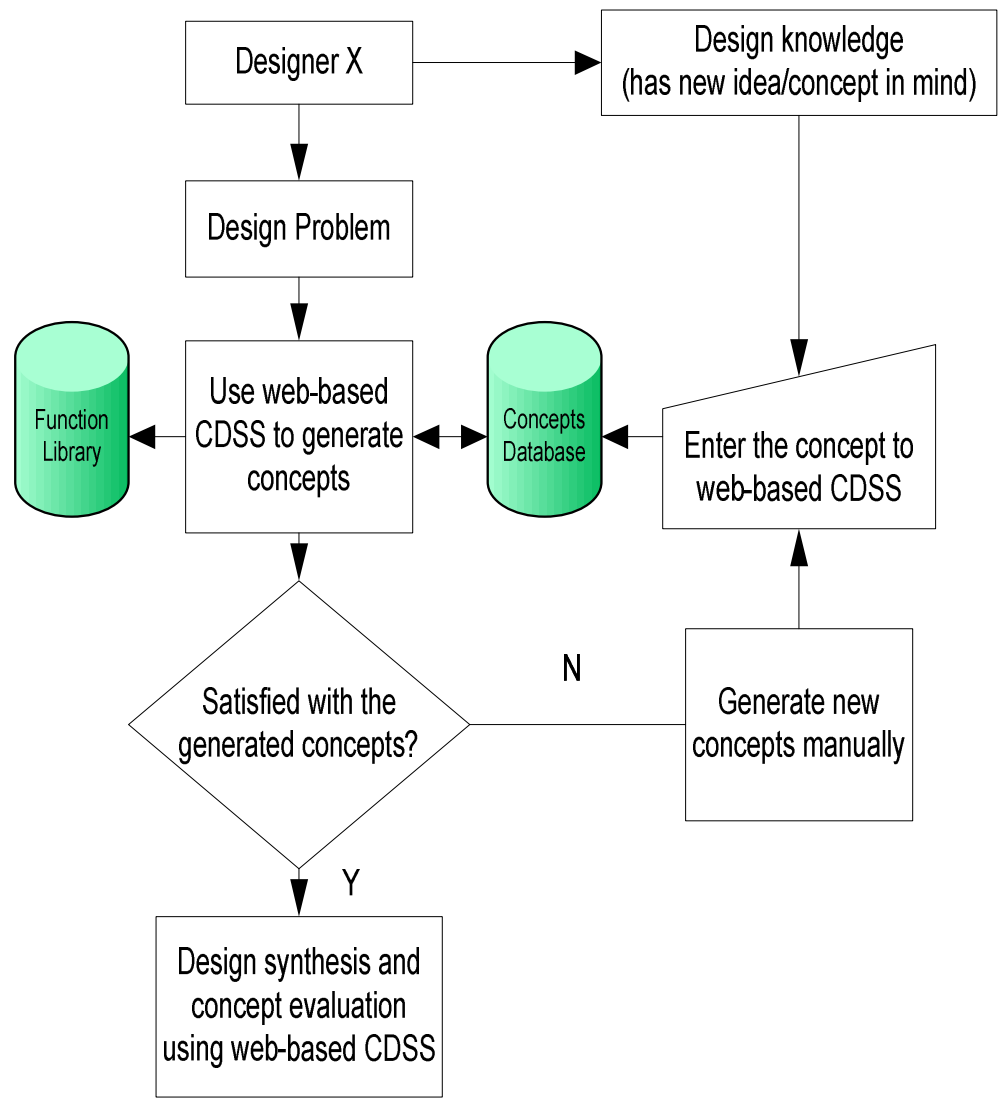

Fig. 1 A framework of the proposed web-based CDSS

\section{Result and Discussions}

The proposed web-based CDSS has been implemented using Django 1.6 [7], which is a high-level Python web framework. The web-based CDSS has been deployed in a local server and can be accessed on intranet network. Each user of the web-based CDSS has to register to use the system and add new concepts to the database. The administrator and designated users review the submitted concepts to be added to the database.

Once a registered user log in to the system, the user can navigate the CDSS through user friendly interface. For instance, to start new design the user has to select the menu which will lead to function library from which the user selects the subfunctions in the functional structure of the specified design task as shown in Fig. 2. The functional structure in the form of subfunctions will be given as input to the design knowledge base system. This is followed by generating concepts from the database. If some of the subfunctions do not have alternative concepts in the database, the designer can generate concepts and give as input to the system for future design. The generated concepts are displayed on the morphology chart where the designer will either accept or reject the concepts based on go-no-go concept selection process.

Under design concepts menu (Fig. 2) there are three submenus i.e., search available concepts, explore existing concepts, and add new concept, which will lead to separate pages. Using explore existing concepts users can review all the concepts with their attributes saved in the database. Exploring all concepts will be difficult and time consuming as the number of concepts in the database increase. Thus, a separate page to search for available concepts is provided. The user can use key words such as primary functions, secondary functions and concept names to search for alternative concepts. 


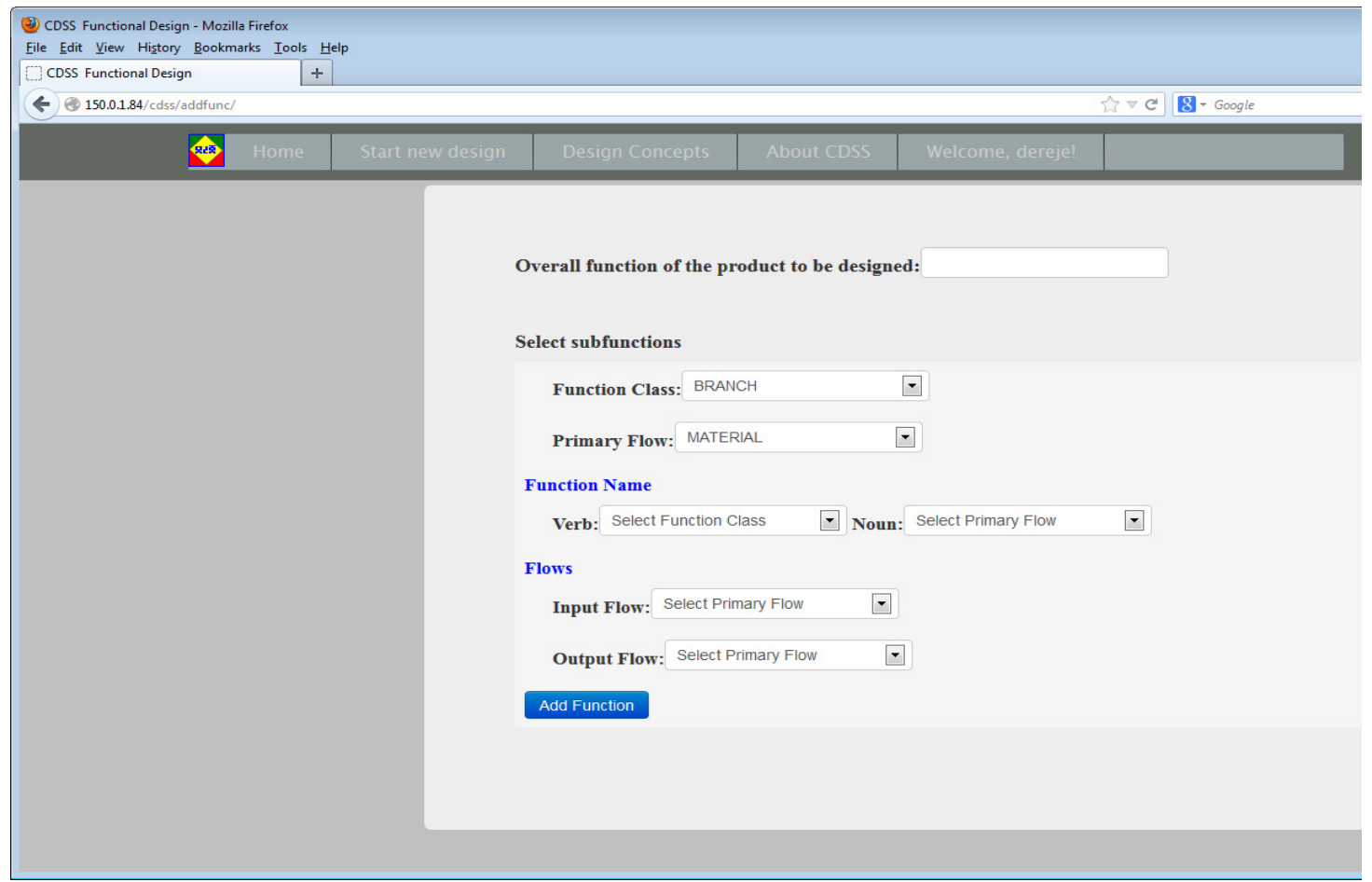

Fig. 2 Function library

The page to add new concepts is shown in Fig. 3. The attributes acquired in adding new concepts to the database include: concept name, primary function, secondary function, input and output flows, sketches and any addition description about the concept. The primary and secondary functions should be described using the vocabulary of functions in the functional basis.

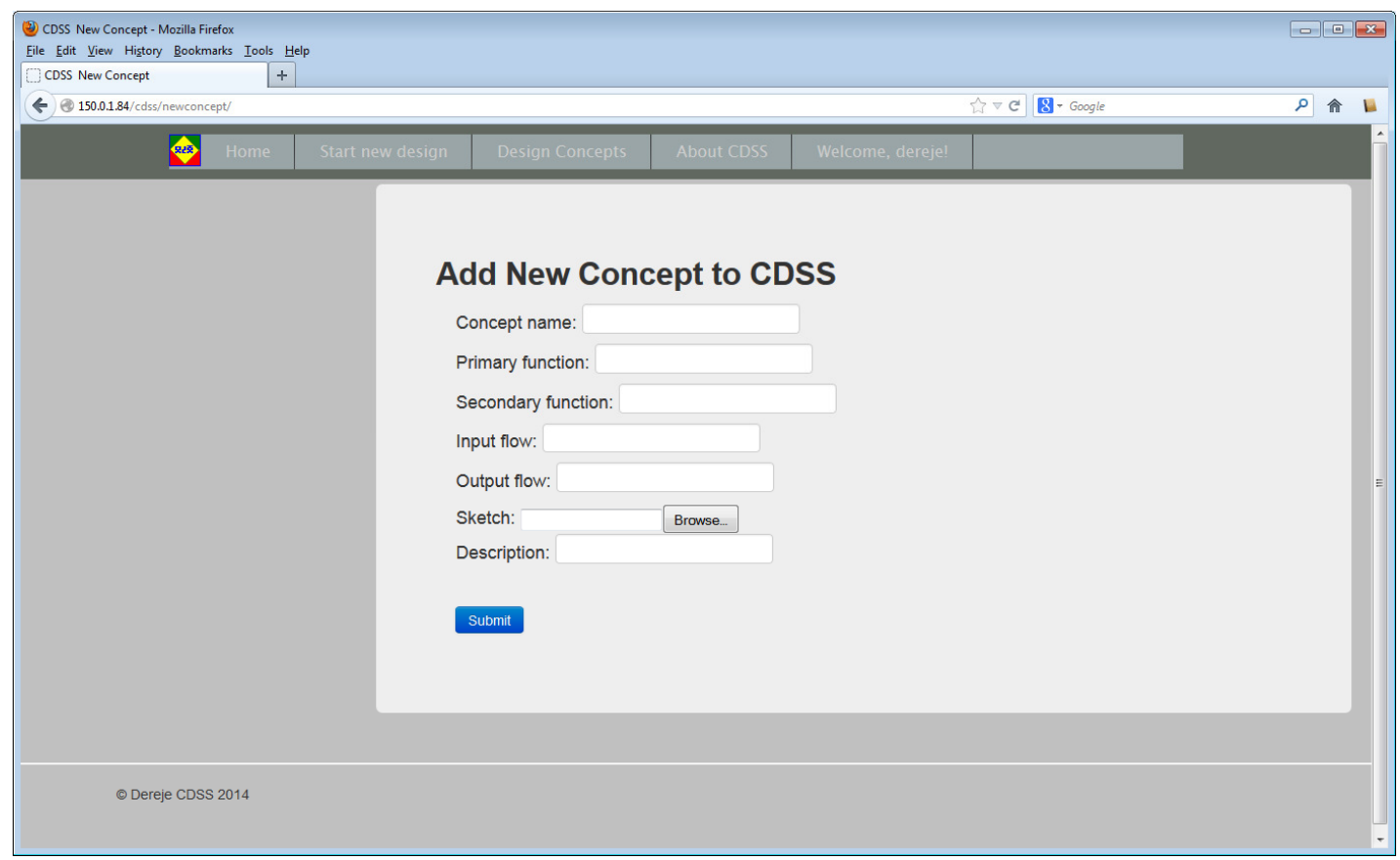

Fig. 3 Attributes of concepts 


\section{Conclusions}

A web-based conceptual design support system has been developed and deployed on an intranet as a collaborative conceptual design support system. CDSS can be used in concept generation process enhancing the designer's knowledge by providing stored knowledge (from design repository) outside the designer's area of expertise. The platform can also be used as a knowledge management system acquiring design knowledge from the designer for future use. The next phase of this work is to build design repository of electro mechanical systems using CDSS platform. The system will be tested using various case studies. The system will be deployed on external web server to be accessed on the World Wide Web.

\section{Acknowledgement}

The authors would like to acknowledge Universiti Teknologi PETRONAS financial support to carry out this project.

\section{References}

[1] Woldemichael, D.E. and F.M. Hashim, Development of conceptual design support tool for subsea process equipment design. International Journal of Mechanical \& Mechatronics Engineering, IJMME, 9(10) 2009. p. 12-17.

[2] Woldemichael, D.E., F.M. Hashim and S. Debnath, A knowledge-based conceptual design support system. Advanced Materials Research, 308 2011. p. 1540-1545.

[3] Woldemichael, D.E. and F.M. Hashim, A framework for function-based conceptual design support system. Journal of Engineering, Design and Technology, 9(3) 2011. p. 250-272.

[4] Tomiyama, T., Intelligent computer-aided design systems: Past 20 years and future 20 years. Artificial Intelligence for Engineering Design, Analysis and Manufacturing, 21 2007. p. 27-29.

[5] Goel, A.K., S. Vattam, B. Wiltgen, and M. Helms, Cognitive, collaborative, conceptual and creative - four characteristics of the next generation of knowledge-based cad systems: A study in biologically inspired design. Computer-Aided Design, 44(10) 2012. p. 879-900.

[6] Hirtz, J., R. Stone, D. McAdams, S. Szykman, and K. Wood, A functional basis for engineering design: Reconciling and evolving previous efforts. Research in Engineering Design, 13(2) 2002. p. $65-82$.

[7] Django Software Foundation, Django: The Web framework for perfectionists with deadlines. Available from: https://www.djangoproject.com/. Accessed in March 2014. 